\title{
Call to Action: Enhancing 'Exercise is Medicine' from the Per- spective of Fitness Professionals
}

\author{
Taniya S Nagpal ${ }^{1,2,3}$, Liza Stathokostas ${ }^{2}$, Harry Prapavessis ${ }^{2,3}$ and Michelle F Mottola ${ }^{1,2,4,5 *}$ \\ ${ }^{1}$ R. Samuel McLaughlin Foundation-Exercise and Pregnancy Laboratory, University of Western Ontario, Canada \\ ${ }^{2}$ School of Kinesiology, Faculty of Health Sciences, University of Western Ontario, Canada \\ ${ }^{3}$ Exercise and Health Psychology Laboratory, University of Western Ontario, Canada \\ ${ }^{4}$ Department of Anatomy and Cell Biology, University of Western Ontario, Canada \\ ${ }^{5}$ Children's Health Research Institute, University of Western Ontario, Canada
}

*Corresponding authors: Dr. Michelle F Mottola, PhD, FACSM, Director, R. Samuel McLaughlin Foundation-Exercise \& Pregnancy Laboratory, University of Western Ontario, London, Ontario, Canada, Tel: 519-661-2111-X88366, Fax: 519661-2008,E-mail: mmottola@uwo.ca

\begin{abstract}
Exercise is Medicine (EIM) is an international initiative that aims to incorporate exercise in primary healthcare by encouraging physicians to prescribe exercise and/or refer patients to Fitness Professionals (FPs). To determine how to increase referral and prescription practices previous research has focused solely on the perspective and recommendations of physicians, not FPs. The purpose of the present pilot study was to engage FPs in a focus group to identify problems and discuss solutions to better implement exercise prescriptions and referrals. Personal trainers $(n=$ 12) were recruited to participate. Using a thematic analysis four themes emerged from the focus group that represent the suggestions of FPs to bridge the gap between FPs and Physicians: (1) Increase communication opportunities between physicians and FPs; (2) Increase EIM educational opportunities for physicians and FPs; (3) Include progression and follow-up on the EIM prescription pad and; (4) Prepare the recreational environment to receive exercise referrals.
\end{abstract}

\section{Keywords}

Exercise is medicine, Exercise prescription, Exercise referrals, Physical activity

\section{Introduction}

Research is now well-established in demonstrating that physical activity can prevent, manage and treat chronic diseases and lower mortality rates [1,2], yet only $10 \%$ of American and $15 \%$ of Canadian adults meet physical activity guidelines of 150 minutes of moderate to vigorous activity per week $[3,4]$. One global health promotion strategy that addresses the issue of inactivity is Exercise is Medicine (EIM). EIM aims to increase physical activity levels of all population groups by promoting the inclusion of exercise prescription and referral in the healthcare system [5,6]; including the use of the EIM Physical Activity Prescription Pad [7]. This referral approach can be direct (i.e., physician consults with the patient) or indirect (i.e., physician refers patient to a fitness professional who then consults with the patient). With respect to the former approach, historic and current exercise prescription by physicians is low with research conducted in this area identifying lack of knowledge [7-14] and lack of time [1,12,15] as being the main barriers to exercise prescription by physicians. EIM envisions and encourages a collaborative approach between healthcare providers and FPs to promote physical activity $[5,6]$, yet the perspective of FPs has not been previously explored. FPs are at the receiving end of the exercise prescription and referral procedure and therefore their perspective and suggestions are an important call to action for EIM to increase exercise prescription and referral practices.

The current pilot study consisted of a convenience sample of FPs from a university campus. The university 
campus is a compelling setting to study the physician-fitness referral as it very often includes health services and recreation centres receiving EIM promotions through their Exercise is Medicine on Campus (EIMC) group. In a focus group setting participants were asked to describe barriers to and enablers of the exercise prescription and referral procedure. It was hypothesized that FPs would primarily discuss the lack of communication between FPs and physicians as this has been previously identified as the main reason physicians choose not to make exercise referrals [16]. As EIM is a relatively recent initiative, and the majority of Promotion and educational opportunities have been mainly directed at physicians, it was hypothesized that FPs would have limited knowledge of EIM. As such, a brief EIM information session was provided prior to the focus group and information on FPs knowledge of EIM was gathered via structured written questionnaires. This study will provide valuable insight for the larger EIM initiative [5] and improve the effectiveness of EIM in its mission to increase the inclusion of exercise in the healthcare system through exercise prescriptions and referrals.

\section{Manuscript Body Text}

\section{Methods}

The pilot study took place at the University of Western Ontario, London, Ontario, Canada (campus population 30,844). Ethics approval was obtained from the Institutional Research Ethics Board. Fourteen certified personal trainers employed at the Western Student Recreation Centre were asked to participate. Participants received an information letter and a written informed consent was obtained to participate in both the introductory presentation and focus group. No personal identifiers were collected.

Before beginning the focus group participants completed a short questionnaire to evaluate their pre-existing knowledge on the EIM goals, mission, resources, and the envisioned role of physicians and role of FPs (Supplementary document 1). The questionnaire was developed with the support of EIM Canada representatives. Following the questionnaire, participants were given a 15 minute standard introductory presentation provided by EIM Canada and delivered by the EIMC graduate student chair. Immediately after the presentation, participants completed a post-information session questionnaire that consisted of the same pre-session questionnaire to evaluate the effectiveness of the information session in improving their knowledge about EIM (Supplementary document 2).

All participants then engaged in one focus group given immediately after the information session. The aim of the focus group was to identify and present suggestions on how to improve the exercise prescription and referral procedure. Participants were informed that the focus group was being recorded and would remain confidential. Focus group questions were open ended and questioned various aspect of EIM (the role of the physician, the role of the FP, effectiveness of exercise prescriptions and referrals in increasing physical activity in the general population).

\section{Statistical analysis}

Questionnaires were scored out of seven (number of correct responses). To determine if there was a change in knowledge from pre to post questionnaires, a paired Student's T-Test was performed and standard deviations were calculated (SPSS version 24).

A thematic analysis was conducted for the focus group using Quirkos 1.3.2. The audio from the focus group was transcribed and transferred to Quirkos. Focus group responses were reviewed and themes were identified. Four themes were identified representing the suggestions from the FPs to improve the exercise prescription and referral procedure. Reasons for the suggestion and associated ideas on how to apply each suggestion were grouped under each theme. Themes were reviewed a second time by two independent investigators who were not involved with the facilitation of the focus group.

\section{Results}

Twelve out of 14 personal trainers $(n=12$, female $=$ 5 , male $=7$, age $=19-24$ years) at the Western Student Recreation Centre participated. Two personal trainers chose not to participate. All personal trainers were graduate or undergraduate students at the institution and certified as a Canadian Society for Exercise Physiology $(n=7)$ or CanFitPro $(n=5)$ personal trainer. Years certified ranged from 1-4 years.

Average score on the pre-information session questionnaire was 1.5 out of 7 ( \pm 1.7 ) or $21 \%$ correct. After the information session, scores significantly improved to an average of 5.75 out of 7 ( \pm 0.75 ) or $82 \%$ correct $(p<0.05)$.

Qualitative thematic analysis for the focus group revealed four major themes or suggestions:

\section{Theme 1: Communication between physicians and FPs}

The first theme was that there was no system in place to allow communication between physicians and FPs and therefore EIM should aim to provide greater opportunities for physicians. Issues identified under this theme included no follow-up system to communicate with a physician about the progress of a patient and no system in place to connect physicians with local FPs. A recommendation to address these concerns was to have FPs employed within health clinics so a patient can be sent directly to them for exercise counselling after their appointment with the general practitioner. A second recommendation was to create an online database that includes the contact information of local FPs for referrals. 


\section{Theme 2: Increase educational opportunities for FPS and physicians}

The next theme identified by the FPs was that there needs to be greater promotion and educational opportunities on EIM to assure all physicians and FPs can participate in the through medical school training or do not know of EIM, this may reduce their confidence in prescribing or referring exercise. A lack of time in appointments was also a concern, if physicians do not spend enough time discussing exercise, then patients may be less likely to adhere to exercise recommendations provided by their physician. Similarly, if FPs are unaware of EIM they will not be prepared to receive an exercise referred patient and cannot participate in the promotion of EIM. Suggestions to improve promotion and education opportunities included: having an exercise representative promote exercise similar to how pharmaceutical companies market new drugs to physicians and increase promotion for the option to refer patients for exercise to FPs rather than focusing physicians primarily on writing an exercise prescription and increase promotion of EIM educational opportunities for FPs as well.

\section{Theme 3: Modifications to the EIM prescription pad}

The third theme was the need to improve the actual prescription pad used in the exercise prescription and subsequent referral. The Frequency, Intensity, Time, Type (FITT) principle was described as helpful by some of the participants but many said there needs to be opportunities available to discuss the prescription with the physician. Furthermore, participants said there was nothing on the current prescription pad about progress (e.g. how long the patient should follow the FITT prescription from the physician before they can increase the intensity, time or change the type of activity). To improve the prescription pad the following suggestions were provided: include a section for progression and develop a follow up system where the physician can Communicate with the FP about the prescription (e.g. online database/system).

\section{Theme 4: Informing the environment about EIM}

The fourth theme focused on educating all staff in a recreational/fitness centre environment to assure EIM is effectively implemented. Participants stated that membership services, front desk personnel, and other employees are all potential individuals that an exercise referred patient may interact with and therefore everyone should be educated on EIM to direct the patient correctly. A final concern related to the gym environment was the issue of cost. All participants felt it was unfair if a patient was given an exercise prescription but also has to purchase a gym membership or pay for a certified FP to meet the recommendations on the prescription. Participants felt that this would decrease motivation to adhere to an exercise prescription or referral. To address these participants recommended EIM should increase advocacy efforts to cover costs associated with referring or prescribing a patient to exercise to assure that cost does not prevent patients from fulfilling their exercise prescription or referral.

\section{Discussion}

The present pilot study is the first to provide the perspective of FPs on the EIM initiative and their recommendations on how to increase exercise prescription and referrals. The current convenience sample of FPs had limited baseline knowledge and awareness of the EIM initiative and that improved significantly from a brief education session. The scores on the pre and post Questionnaires in the present study suggest that EIM training and educational opportunities are beneficial and needed for FPs.

A thematic analysis of the focus group revealed four themes regarding barriers to and facilitators of exercise prescription and the referral procedure from the perspective of FPs. Firstly, as hypothesized; FPs expressed the need to improve communication between the referring physician and FPs. Two qualitative studies from the perspective of physicians also had the same finding where physicians felt that there is no system in place to connect with FPs and this was a reason as to why they do not refer patients for exercise [1,2]. Current literature does not suggest any consensus on the recommended intensity of a FPs involvement for implementing an exercise prescription however, in our study, FPs stated that to improve the referral process and to assure the patient does seek professional support to meet their exercise goals there should be a way to engage with physicians and discuss the patient's initiation of exercise goals and their ongoing progress. Follow up support from healthcare providers has been identified as an effective strategy to increase compliance to exercise recommendations [6] and by implementing an online system for FPs and physicians to communicate with one another and including FPs in the medical system, there may be greater opportunity to provide patients with effective feedback and support to meet their exercise prescription. There are existing resources available for online communication between FPs and physicians $[17,18]$ however it is unknown if all fitness professionals and physicians are aware of these tools and are available through the database. Perhaps EIM can increase promotion for already available tools or advocate for the development of a universal online portal that allows for reciprocal communication between the FP and physician and assure that this is implemented in all clinical and recreational settings.

FPs stated that the lack of education physicians have regarding exercise prescription and the benefits of exercise are concerning and this may be why physicians do not prescribe exercise. Currently EIM has focused primarily on equipping physicians with the tools to prescribe exercise $[1,7,8,10,12,17]$ however by emphasiz- 
ing the importance of exercise referrals, EIM may be able to engage FPs more and support physicians who may lack the knowledge to counsel or prescribe patients with exercise.

The third area identified for improvement was the prescription pad used to prescribe or refer exercise. This links back to the need for implementing a system to allow communication between physicians and FPs. Studies have found that physicians are hesitant to refer patients for exercise to FPs because they do not have any way to follow up on the patient's progress $[13,16]$. By including a progression and follow up section on the prescription pad, EIM can close the communication gap between FPs and physicians.

Finally, FPs stated that if staff at a recreational facility is unaware of EIM, a patient that with a prescription may be met with surprise. This could potentially decrease their motivation to be physically active and follow through with their referral or prescription. In the present study, all participants were personal trainers because in a gym environment personal trainers are the most likely employees to have one on one interaction with patients. However, it is possible a patient prescribed to exercise may inquire about EIM from any recreational centre staff member, therefore all recreational staff may benefit from EIM education sessions. Furthermore, FPs emphasized the need to consider costs associated with being referred to a gym and/or a personal trainer. Cost has been identified as a barrier to physical activity [19-21]. Unlike medication, gym memberships and personal trainer costs are not covered by health insurance and this may decrease the likelihood that a patient will follow through with their exercise prescription or referral. If FPs and physicians are engaged with EIM and exercise is included in the healthcare system, then perhaps advocacy efforts towards considering costs associated with exercise prescriptions and referrals will be implemented.

Studies have examined how to improve the exercise prescription and referral procedure from the perspective of physicians $[9,11,12,16,17]$, yet EIM believes both physicians and FPs are key members in promoting and implementing the inclusion of exercise in the healthcare system [5]. By conducting a focus groups with personal trainers employed at a campus recreation facility, we are the first pilot study to document information from the perspective of FPs. The suggestions provided by FPs inform EIM with an action plan to improve the exercise prescription and referral procedure.

\section{Conclusion}

The results of the current pilot study suggest a call to action for EIM. To improve the exercise prescription and referral procedure FPs recommended: 1) An increase in communication opportunities between FPs and physicians through online resources; 2 ) More opportunities to promote and educate physicians and FPs on EIM; 3) The exercise prescription pad should be improved to include more detail about follow up and progression, and finally; 4) Recreation settings need to be improved by educating all staff about EIM and costs associated with prescribing exercise at a gym should be considered. These findings are a stepping stone towards better understanding the perspective of FPs and to inform EIM with suggestions that can potentially enhance the effectiveness of the EIM initiative to increase physical activity levels in all population groups. Limitations of the current pilot study include the lack of validated pre- and post-presentation questionnaires and a small sample size however future work can utilize the questionnaires developed for this pilot study to validate them for evaluating the knowledge FPs have on EIM. Furthermore, future work should include a more diverse sample, including participants from other fitness professions, varying years of experience and employed at different recreational facilities, and consider a mixed approach exploring the perspective of both physicians and FPs together to expand on current findings.

\section{Conflict of Interest Statement}

All authors declare no conflict of interest.

\section{Financial Disclosures}

All authors declare no financial disclosures.

\section{References}

1. Pedersen BK, Saltin B (2015) Exercise as medicine: Evidence for prescribing exercise as 242 therapy in 26 different chronic diseases. Scandinavian Journal of Medicine and Science in Sports 25: 1-72.

2. Warburton DE, Nicol CW, Bredin SS (2006) Health benefits of physical activity: the evidence. CMAJ 174: 801-809.

3. Haskell WL, Lee I, Pate RR, Powell KE, Blair SN, et al. (2007) Physical activity and public health: Updated recommendation 248 for adults from the American College of Sports Medicine and the American Heart Association. Circulation 116: 1081-1093.

4. Tucker JM, Welk GJ, Beyler NK (2011) Physical activity in U.S.: adults compliance with the Physical Activity Guidelines for Americans. Am J Prev Med 40: 454-461.

5. Sallis RE (2009) Exercise is medicine and physicians need to prescribe it! Br J Sports Med 43: 3-4.

6. Sallis R, Franklin B, Joy L, Ross R, Sabgir D, et al. (2015) Strategies for promoting physical activity in clinical practice. Prog Cardiovasc Dis 57: $375-386$.

7. Frémont $\mathrm{P}$, Fortier $\mathrm{M}$, Frankovich RJ (2014) Exercise prescription and referral tool to facilitate brief advice to adults in primary care. Can Fam Physician 60: 1120-1122, e591-e592.

8. Abramson S, Stein J, Schaufele M, Frates E, Rogan S (2000) Personal exercise habits and counseling practices of primary care physicians: a national survey. Clin J Sport Med 10: 40-48.

9. Heath GW, Kolade VO, Haynes JW (2015) Exercise is medicine: A pilot study linking 263 primary care with community physical activity support. Prev Med Rep 2: 492-497. 
10. Kennedy MF, Meeuwisse WH (2003) Exercise counselling by family physicians in Canada. Prev Med 37: 226-232.

11. Persson G, Brorsson A, Ekvall Hansson E, Troein M, Strandberg EL (2013) Physical activity on prescription (PAP) from the general practitioner's perspective - a qualitative study. BMC Fam Pract 14: 128.

12. Petrella RJ, Lattanzio CN, Overend TJ (2007) Physical activity counseling and prescription among canadian primary care physicians. Arch Intern Med 167: 1774-1781.

13. Williams $\mathrm{NH}$, Hendry $M$, France $B$, Lewis $R$, Wilkinson $C$ (2007) Effectiveness of exercise-274 referral schemes to promote physical activity in adults: Systematic Review. $\mathrm{Br}$ J Gen Pract 57: 979-986.

14. Windt J, Windt A, Davis J, Petrella R, Khan K (2015) Can a 3-hour educational workshop and the provision of practical tools encourage family physicians to prescribe physical activity as medicine? A pre-post study. BMJ Open 5: e007920.
15. Walsh JM, Swangard DM, Davis T, McPhee SJ (1999) Exercise counseling by primary care physicians in the era of managed care. Am J Prev Med 16: 307-313.

16. Graham RC, Dugdill L, Cable NT (2005) Health professionals' perspectives in exercise referral: implications for the referral process. Ergonomics 48: 1411-1422.

17. ACE Pro (2017) Find an ACE certified professional.

18. Idea Health and Fitness Association (2017) Search for a fitness professional.

19. Hill LL, Nichols J, Wing D, Waalen J, Friedman E (2015) Training on exercise is medicine ${ }^{\circledR}$ within an integrative medicine curriculum. Am J Prev Med 49: S278-S284.

20. Salmon J, Owen N, Crawford D, Bauman A, Sallis JF (2003) Physical activity and sedentary behavior: a population-based study of barriers, enjoyment, and preference. Health Psychol 22: 178-188.

21. Tappe MK, Duda JL, Ehrnwald PM (1989) Perceived barriers to exercise among adolescents. J Sch Health 59: 153-155. 


\section{Supplementary Document 1:}

\section{Exercise as a Vital Sign on Campus: Pre-Information} Session Questionnaire

Please circle the correct answer, please do not guess. If you do not know an answer or do not wish to answer a question please circle option $D(N / A)$.

1. What is the mission of Exercise is Medicine Canada?

a. To increase the number of physicians prescribing exercise to their patients.

b. To increase the number of Canadians achieving the daily Canadian Physical Activity Guidelines.

c. To include physical activity in the Canadian Healthcare System as a prevention and treatment method for chronic diseases.

d. N/A

2. Which two key individual groups are involved in the prescribing and referral process of exercise?

a. Physicians and Patients.

b. Exercise Professionals and Physicians.

c. Exercise Professionals and Patients.

d. $N / A$

3. Exercise is Medicine is encouraging what change in our Canadian Healthcare System?

a. Accept exercise as a form of medicine to be prescribed.

b. Increase counselling, prescribing and referrals of exercise from physicians.

c. To replace over the counter medication and prescription medicine with exercise first.

d. N/A
4. In order to prescribe exercise, what tool has Exercise is Medicine created and based on what principle can it be utilized to create an exercise prescription?

a. Online learning module; FITT Principle.

b. Smart phone application; Personalized Fitness Principle.

c. Prescription Pad; FITT Principle.

d. N/A

5. What role does Exercise is Medicine envision for exercise professionals in this initiative?

a. To receive referrals from patients who have been prescribed exercise and create an exercise prescription for them based on their needs.

b. To refer patients to physicians to be screened for the ability to exercise to help treat or prevent chronic diseases.

c. To work with physicians to introduce exercise as a prescription to prevent and treat chronic diseases.

d. N/A

6. According to Exercise is Medicine, who should be able to prescribe exercise?

a. Physician only.

b. Exercise Professional only.

c. Both Physician and Exercise Professional.

d. N/A

7. As a personal trainer, what would be your key role according to Exercise is Medicine?

a. Receive exercise referrals from physicians.

b. Create an exercise prescription for patients referred to exercise.

c. Can be both A and B.

d. N/A 


\section{Supplementary Document 2:} Exercise as a Vital Sign on Campus: Post-Information
Session Questionnaire

Please circle the correct answer, please do not guess. If you do not know an answer or do not wish to answer a question please circle option $D(N / A)$.

1. What is the mission of Exercise is Medicine Canada?

a. To increase the number of physicians prescribing exercise to their patients.

b. To increase the number of Canadians achieving the daily Canadian Physical Activity Guidelines.

c. To include physical activity in the Canadian Healthcare System as a prevention and treatment method for chronic diseases.

d. $N / A$

2. Which two key individual groups are involved in the prescribing and referral process of exercise?
a. Physicians and Patients.
b. Exercise Professionals and Physicians.
C. Exercise Professionals and Patients.
d. N/A

3. Exercise is Medicine is encouraging what change in our Canadian Healthcare System?
a. Accept exercise as a form of medicine to be pre- scribed.
b. Increase counselling, prescribing and referrals of ex- ercise from physicians.
c. To replace over the counter medication and prescrip- tion medicine with exercise first.

\section{d. N/A}

4. In order to prescribe exercise, what tool has Exercise is Medicine created and based on what principle can it be utilized to create an exercise prescription?
a. Online learning module; FITT Principle.
b. Smart phone application; Personalized Fitness Prin-

ciple.

C. Prescription Pad; FITT Principle.

d. $N / A$

5. What role does Exercise is Medicine envision for exercise professionals in this initiative?

a. To receive referrals from patients who have been prescribed exercise and create an exercise prescription for them based on their needs.

b. To refer patients to physicians to be screened for the ability to exercise to help treat or prevent chronic diseases.

c. To work with physicians to introduce exercise as a prescription to prevent and treat chronic diseases.

d. N/A

6. According to Exercise is Medicine, who should be able to prescribe exercise?
a. Physician only.
b. Exercise Professional only.
c. Both Physician and Exercise Professional.
d. $N / A$

7. As a personal trainer, what would be your key role according to Exercise is Medicine?

a. Receive exercise referrals from physicians.

b. Create an exercise prescription for patients referred to exercise.

C. Can be both $A$ and $B$.

d. N/A

8. Please rank the information session you received in its helpfulness in increasing your knowledge about Exercise is Medicine.

$\begin{array}{llllll}0 & 1 & 2 & 3 & 4 & 5\end{array}$

N/A Not very A little No difference Helpful Very Helpful Helpful Helpful 